\title{
Self-Reported Utilization of Preventive Health Services by Retired Employees Age 65 and Older
}

\author{
Shirley Musich, PhD, * Aartee Ignaczak, MPH, * Timothy McDonald, MHSA, ${ }^{\dagger}$ \\ David Hirschland, MBA, and Dee W. Edington PhD*
}

\begin{abstract}
OBJECTIVES: Increased utilization of preventive services among the aging has been associated with improved health status and decreased medical costs. We sought to examine the use of the Health Risk Appraisal (HRA) in benchmarking compliance and characterizing those retired employees who met preventive service guidelines.

DESIGN: A retrospective cohort study of retired employees age 65 and older.
\end{abstract}

SETTING: Nation-wide health promotion program offered by General Motors Corporation.

PARTICIPANTS: 59,670 retired General Motors employees age 65 and older who participated in a nationwide mailed HRA health promotion program.

MEASUREMENTS: Preventive health services compliance was measured using selected HRA questions. Gender, HRA participation patterns, overall health risk status, medical plan selection and disease status were examined as predictors of increased compliance. Multivariate logistic regression models were developed to test the relative contributions of participant characteristics to increased utilization. RESULTS: The self-reported HRA data indicated that compliance levels were higher than national averages. The Healthy People 2000 goals for the preventive services studied were met and exceeded (with the exception of tetanus immunization). Higher compliance was associated with being male, younger than 70 years, multiple-year HRA participation, overall low risk status and HMO insurance plan selection.

CONCLUSION: The results from the HRA indicated that this population participated at a higher level than a comparable national sample exceeding goals set by Healthy People 2000. J Am Geriatr Soc 49:1665-1672, 2001.

Key words: preventive services; HRA; retired employees

From the "Health Management Research Center, University of Michigan, Ann Arbor, Michigan; ${ }^{\dagger}$ General Motors Corporation, Detroit, Michigan; and International Union, United Automobile, Aerospace and Agricultural Implement Workers of America, Detroit, Michigan.

Address correspondence to D. W. Edington, PhD, Health Management Research Center, University of Michigan, 1027 E. Huron Street, Ann Arbor, MI 48104.
Uuccessful aging, characterized by continued good health, lack of disease and maintenance of functional status, is a desirable goal among the retired population. Recently, health promotion activities for older people have focused on behavioral health risks such as exercise habits, smoking, and weight management and chronic conditions like hypertension. Personalized Health Risk Appraisals (HRAs) have been used among retirees to increase awareness of personal health and to promote favorable changes in individual health habits. ${ }^{1}$ An additional purpose of the HRA has focused on the assessment of utilization of preventive services and early detection of disease for the maintenance of health. The HRA has been used to monitor changes in behavioral health risks but little attention has been given to using the HRA to increase awareness of preventive services and to monitor utilization of preventive services.

Participation in preventive health screenings among Medicare-eligible persons was shown to increase during a preventive services intervention. ${ }^{2}$ Participants in the intervention group were offered a free preventive services "package." Control group participants received a booklet on prevention and maintaining good health. Of the individuals in the intervention group, $63 \%$ made the initial preventive visit after the baseline interview. After 2 years, the intervention group showed a greater health benefit and a significantly lower death rate compared with the control group. Contrary to expectations, the increased preventive services did not contribute to increased Medicare charges but did contribute to a reduced number of ambulatory visits and lower hospital charges. ${ }^{3}$ In a follow-up study 2 years after the intervention, the differences in preventive services use and the improvement in health status were not maintained. ${ }^{4}$ The authors suggested that out-of-pocket costs for preventive services may have been a barrier to continued use of preventive services. In addition, patients were not educated as part of the original intervention program about the importance of continuing to use preventive services.

A parallel study by Morrissey et al. ${ }^{5}$ demonstrated that preventive services utilization could be increased significantly in a controlled intervention. At 2-year follow-up, there were minimal differences in health-related qualityof-life indicators between intervention and control groups. However, consistent with the previous study, actual Medicare costs for the intervention group were less than for the 
control group with significant savings in hospital utilization over the 2 years.

The HRA has been shown to be an effective tool for raising awareness about health behaviors in the short term. ${ }^{6,7}$ However, the question arises as to whether the increased awareness resulting from HRA participation leads to increased compliance of preventive health services over a number of years. Among the population age 65 and older, barriers to compliance include a complex mix of medical coverage limitations, lack of awareness, fear of positive screening results, physical access problems and inconsistency in guidelines for preventive services compliance. ${ }^{8}$

The purposes of this study were to use the HRA as a measurement tool in benchmarking preventive services compliance among a retired employee population, compare their utilization of preventive services with national goals, and describe selected participant characteristics that may be predictive of increased compliance.

\section{METHODS}

This study focused on the retired employees from General Motors Corporation (GM) who were 65 years or older as of January 1996, and who resided in the United States. Retired employees included members of the International Union, United Automobile, Aerospace and Agricultural, Implement Workers of America.

As part of the company-wide health promotion program, an annual HRA was mailed to each employee. The completed HRAs were returned to the University of Michigan Health Management Research Center for processing. An individualized health profile was then mailed directly to the individual. A quarterly health newsletter, self-care book and 1-800 health information phone service was also available as part of the health promotion program. The study population consisted of 59,670 retired employees who completed at least one HRA during the 3-year time period 1996 to 1998 (participation rate, 39\%). Spouses of employees and/or dependents were not included in the study group.

The HRA questionnaire collected demographic information and information on health-related behaviors, quality of life indicators and medical history/self care, which included questions regarding current practices related to preventive health service utilization. The specific preventive service information obtained via the questionnaire pertained to influenza and tetanus immunization, blood pressure, and cholesterol screening and colorectal cancer screening. Additionally, information was obtained regarding gender specific preventive services such as mammogram screenings and Papanicolaou (Pap) tests for women and prostate examinations for men.

Compliance with preventive services for each analysis grouping was determined based on the guidelines set forth in the U.S. Preventive Services Task Force publication "Guide to Clinical Preventive Services, 2nd edition." 9 The specific question on the HRA questionnaire which addressed preventive services was: "When was the last time you had these preventive services or health screenings?" with the available responses: "less than 1 year ago, 1-2 years ago, 3-4 years ago, 5 or more years ago, never or don't know." Because these year intervals did not always correspond to the Clinical Preventive Services guidelines, some adjustments were necessary in establishing cut-points for the analyses of preventive services compliance. The cut-points used for each preventive service are given in Table 1.

Table 1. Clinical Preventive Service Guidelines

\begin{tabular}{|c|c|c|}
\hline Preventive Service & $\begin{array}{l}\text { Clinical Preventive Service } \\
\text { Guideline }^{5}\end{array}$ & $\begin{array}{l}\text { Guidelines Used } \\
\text { for Analysis }\end{array}$ \\
\hline Influenza immunization & $\begin{array}{l}\text { Individuals } \geq 65 \\
\text { Annually }\end{array}$ & $\begin{array}{l}\text { Individuals } \geq 65 \\
<1 \text { year }\end{array}$ \\
\hline Blood pressure screening & $\begin{array}{l}\text { Individuals } \geq 18 \\
\leq 2 \text { years }\end{array}$ & $\begin{array}{l}\text { Individuals } \geq 65 \\
\leq 2 \text { years }\end{array}$ \\
\hline Cholesterol screening & $\begin{array}{l}\text { Men age } 35-65 \\
\text { Women age } 45-65 \\
\text { Periodic screening }\end{array}$ & $\begin{array}{l}\text { Individuals } \geq 65 \\
\leq 4 \text { years }\end{array}$ \\
\hline Tetanus immunization & $\begin{array}{l}\text { Vaccine for all adults } \\
\text { Once every } 10 \text { years }\end{array}$ & $\begin{array}{l}\text { Individuals } \geq 65 \\
\geq 5 \text { years }\end{array}$ \\
\hline $\begin{array}{l}\text { Colon cancer screen (via } \\
\text { sigmoidoscopy or FOBT) }\end{array}$ & $\begin{array}{l}\text { Individuals } \geq 50 \\
\text { FOBT } \leq 1 \text { year } \\
\text { Sigmoidoscopy }=3-5 \text { years. }\end{array}$ & $\begin{array}{l}\text { Individuals } \geq 65 \\
\leq 4 \text { years }\end{array}$ \\
\hline Rectal examination & $\begin{array}{l}\text { Individuals } \geq 18 \\
\text { Annually }\end{array}$ & $\begin{array}{l}\text { Individuals } \geq 65 \\
<1 \text { year }\end{array}$ \\
\hline Breast cancer screening & $\begin{array}{l}\text { Women age } 50-69 \\
\leq 2 \text { years }\end{array}$ & $\begin{array}{l}\text { Women } \geq 65 \\
\leq 2 \text { years }\end{array}$ \\
\hline Cervical cancer screening & $\begin{array}{l}\text { Sexually active women } \\
\leq 3 \text { years }\end{array}$ & $\begin{array}{l}\text { Women } \geq 65 \\
\leq 4 \text { years }\end{array}$ \\
\hline Prostate cancer screening & No guideline given & $\begin{array}{l}\text { Men } \geq 65 \\
\leq 2 \text { years }\end{array}$ \\
\hline
\end{tabular}

FOBT $=$ fecal occult blood test. 
Overall compliance rates were determined by measuring the number of individuals who met the guidelines for the selected preventive service among those who participated in the HRA questionnaire.

A national database of retired 65 years and over population available from the National Health Interview Survey (NHIS), Clinical Preventive Services supplement, 1994 was used to benchmark compliance levels of the study group. ${ }^{10}$ The NHIS is a national cross-sectional probability sample of the U.S. civilian population. The inclusion criteria of being 65 years and over, of no longer being in the labor force and of having completed the NHIS section on clinical preventive services was met by 3,576 NHIS respondents. Healthy People 2000 goals were also included for comparison. ${ }^{11}$

Additional analyses examined gender, insurance plan selection (indemnity/preferred provider organization (PPO) or health maintenance organization (HMO)), HRA participation patterns, overall health risk status and the existence of medical problems as participant characteristics that could potentially influence overall compliance. Gender and insurance plan selections were obtained from the personnel department of the manufacturing company.

Frequency of participation in the HRA and its possible relationship to compliance was considered by grouping HRA participants into the following categories: three-time HRA participants (annual participation in each of the 3 years); two-time HRA participants (participation in any 2 years); and one-time HRA participants (participation in any 1 year only). It was expected that multiple-year HRA participation would be associated with increased preventive service utilization over time.

Overall health risk level was determined from the HRA by assessing whether an individual had any of the following individual health risks: lack of exercise, smoking, overweight, alcohol use, high blood pressure, high cholesterol, stress, life dissatisfaction, poor perception of health, presence of medical problems or absence due to illness. An overall low-risk level was defined by the presence of any zero to two risks, medium-risk by the presence of any three to four risks and high-risk by the presence of any five or more risks.

An individual was categorized as having a medical problem (disease) if they self-reported on the HRA one or more of the following diseases: cancer, diabetes mellitus, bronchitis/emphysema, heart disease, or previous stroke. For the purposes of our analysis, individuals were categorized by the presence of single disease, having multiple diseases or by the absence of any of the diseases.

Comparisons were tested for statistical significance using chi-square analyses for categorical variables and the analysis of variance procedure for continuous variables. Continuous multi-level comparisons were further tested using post hoc Tukey's Standardized Range Test.

A multivariate logistic regression model was used to test the relative strengths of the selected predictors of preventive service utilization. Dichotomous variables for the regression model were coded as follows: female/male (1/0); age greater than 69 years/less than 70 years $(1 / 0)$; plan selection HMO/indemnity-PPO (1/0); high-risk status $(\geq 3$ risks)/low-risk status (1/0); HRA participation two or more times/1-time (1/0); heart disease/no heart disease (1/0); can- cer/no cancer (1/0); diabetes mellitus/no diabetes mellitus (1/0); bronchitis/no bronchitis (1/0); and stroke/no stroke $(1 / 0)$. A stepwise logistic regression procedure provided final models predicting utilization for each preventive service. Odds ratios (ORs) and confidence intervals for predictor variables were calculated.

\section{RESULTS}

The 59,670 retirees age 65 and older included in the analysis were $60 \%$ hourly and $40 \%$ salaried former employment status with an average age of 74.1 years. Their medical plan selections were $80 \%$ indemnity/PPO and $20 \%$ HMO. The percentage of males and females $(85 \%$ and $15 \%$, respectively) were almost the same in each of these subgroups. The NHIS comparison population consisted of 3,576 individuals 65 years and over who were not in the workforce. It was not possible to determine prior employment status or medical plan selection from the national survey questions. This NHIS group was $34 \%$ male and $66 \%$ female with an average age of 74.9 years.

\section{INFLUENZA IMMUNIZATION}

The recommendation of the U.S. Preventive Services Task Force for influenza immunization is for individuals 65 and older to be immunized annually. ${ }^{9}$ Healthy People 2000 objectives had set a goal of $60 \%$ for ages 65 and older to receive influenza vaccine. ${ }^{11}$ In the current study, $70 \%$ had been immunized for influenza in the past year (Table 2). Data from the 1994 NHIS indicated that $55 \%$ of surveyed retired individuals had been immunized for influenza in the past year.

\section{BLOOD PRESSURE SCREENING}

The U.S. Preventive Services Task Force recommends blood pressure screening for all adults, regardless of age. No time interval for screening is given. The American Heart Association recommends that individuals have their blood pressure checked at least once every 2 years. ${ }^{9}$ Healthy People 2000 objectives had set a goal of $90 \%$ for adults who have had their blood pressure measured in the preceding 2 years. ${ }^{11}$ Among the retirees in our study population, $92 \%$ reported having had their blood pressure checked within the recommended time period (Table 2). In the NHIS sample, 93\% of retirees had had their blood pressure screened within the past 2 years.

\section{CHOLESTEROL SCREENING}

The Guide to Clinical Preventive Services recommends periodic screening for blood cholesterol for men age 35 to 65 and for women age 45 to 65 . No recommendation is made for or against screening in individuals over age 65 . The National Cholesterol Education Program Adult Treatment Panel II, convened by the National Heart, Lung and Blood Institute, recommends that all adults over the age of 20 should have their blood cholesterol screened at least once every 5 years. ${ }^{9}$ In addition, Healthy People 2000 objectives had set a goal of $75 \%$ for adults to be screened for cholesterol within the last 5 years. ${ }^{11}$ In this HRA, response choices for cholesterol screening were "less than 1 year, 1-2 years, 3-4 years, or 5 or more years ago." In evaluating compliance for cholesterol, we used the guideline of 4 years or less. Among the individuals in our study popula- 
Table 2. Preventive Service Compliance: Male vs Female and Indemnity/PPO vs HMO

\begin{tabular}{|c|c|c|c|c|c|c|}
\hline \multirow[b]{3}{*}{ Preventive Service } & \multirow[b]{2}{*}{$\begin{array}{l}\text { Healthy People } \\
2000 \text { Goals }\end{array}$} & \multirow{2}{*}{$\begin{array}{c}\text { Compliance } \\
\text { Overall } \\
(n=59,670)\end{array}$} & \multicolumn{2}{|c|}{ Compliance } & \multicolumn{2}{|c|}{ Compliance } \\
\hline & & & $\begin{array}{c}\text { Male } \\
(n=50,612)\end{array}$ & $\begin{array}{c}\text { Female } \\
(n=9,058)\end{array}$ & $\begin{array}{c}\text { Indemnity/PPO } \\
(\mathrm{n}=47,948)\end{array}$ & $\begin{array}{c}\text { HMO } \\
(n=11,627)\end{array}$ \\
\hline & \multicolumn{6}{|c|}{$\%$} \\
\hline Influenza immunization ( $<1$ year) & 60 & 70 & 70 & $66^{*}$ & 69 & $73^{*}$ \\
\hline Tetanus immunization ( $\geq 5$ years) & $\begin{array}{c}62 \\
(\leq 10 \text { years })\end{array}$ & 54 & 56 & $43^{*}$ & 53 & $60^{*}$ \\
\hline Colon cancer screening ( $\leq 4$ years) & $\begin{array}{c}50 \\
(\leq 2 \text { years) }\end{array}$ & 62 & 64 & $51^{*}$ & 61 & $66^{*}$ \\
\hline Rectal examination ( $<1$ year) & 40 & 48 & 51 & $34^{*}$ & 47 & $52^{*}$ \\
\hline
\end{tabular}

${ }^{*}$ Chi-square $P<.001$.

$\mathrm{PPO}=$ preferred provider organization $; \mathrm{HMO}=$ health maintenance organization.

tion, $83 \%$ had had their cholesterol checked within the past 4 years (Table 2 ).

Among the NHIS sample, $58 \%$ of retirees had had their cholesterol checked within the past 4 years. (Answer choices on the NHIS were limited to " 4 years or less" and "over 4 years.") The question in the NHIS for cholesterol was also linked to "things checked during last check-up" hence screening compliance rates would be expected to be somewhat lower than if screening were not part of a physician's visit.

\section{TETANUS IMMUNIZATION}

The nationally recognized guidelines for immunization against tetanus are for individuals to be immunized once every 10 years. ${ }^{9}$ Healthy People 2000 objectives indicated a goal for $62 \%$ of adults to have received a tetanus booster within the last 10 years. ${ }^{11}$ In the NHIS sample, $27 \%$ of retired individuals reported having complied with this recommendation. Although the national recommendation is for tetanus immunization every 10 years, the HRA used for the current study asked only whether the individual had been immunized in the past 5 or more years. In our study population, $54 \%$ reported having been immunized against tetanus within the past 5 or more years (Table 2).

\section{COLON CANCER SCREENING}

National guidelines recommend that individuals over the age of 50 be annually screened for colorectal cancer by fecal occult blood testing. A separate recommendation of 3 to 5 years is presented for screening by sigmoidoscopy. ${ }^{9}$ The HRA used to collect data for this study asked whether the individual had been screened for colorectal cancer but did not specify the method of screening. Thus, for the purposes of our analysis, we have used a screening guideline of every 3 to 4 years. This 4 -year guideline encompasses individuals who have had a fecal blood test and/or the sig- moidoscopy. Healthy People 2000 goals were for $50 \%$ of those adults age 50 and older to have received fecal occult blood testing within the preceding 2 years and for $40 \%$ to have ever received sigmoidoscopy. ${ }^{11}$ No specific target was given for those age 65 and older.

Among the subjects in our population, $62 \%$ reported having been screened for colorectal cancer in the past 4 years (Table 2). No data were collected from the participants in the NHIS regarding colorectal cancer screening.

\section{COLORECTAL CANCER SCREEN BY DIGITAL RECTAL EXAMINATION}

National guidelines suggest that individuals age 50 and older should be screened for colorectal cancer via digital rectal examination annually. ${ }^{9}$ Healthy People 2000 goals were for $40 \%$ of those 50 and over to have been examined during the past year. ${ }^{11}$ This recommendation was met by $48 \%$ of the subjects in our study population (Table 2 ). No data were collected in the NHIS sample.

\section{BREAST CANCER SCREENING}

National guidelines recommend that women ages 50 to 69 undergo mammography screenings for breast cancer every 1 to 2 years. ${ }^{9}$ Healthy People 2000 goals were for $60 \%$ of women age 50 and older to have mammograms in the preceding 1 to 2 years. This Healthy People goal was extended to include women who were age 70 and older. ${ }^{11}$ Because Healthy People 2000 included all women age 50 and older in their goals, for the purposes of our analyses, we have extended the clinical guidelines to include all of the 9,058 women in our study population. Among these women, $76 \%$ reported they had been screened for breast cancer in the past 2 years (Table 2). In the national group, among the 2,353 females, $51 \%$ had had a mammogram within the last 2 years. 


\section{CERVICAL CANCER SCREENING}

The U.S. Preventive Services Task Force recommends that sexually active women 18 years and over with an intact cervix be screened for cervical cancer every 3 years. ${ }^{9} \mathrm{Al}-$ though Healthy People 2000 goals were for $85 \%$ of women 18 and over to have had a Pap test in the last 3 years, the goal for women 70 years and over was dropped to $70 \% .{ }^{11}$ The HRA utilized in this study asked whether women have been screened "less than 1 year," "1-2 years" or " $3-4$ years ago." Hence, for the 9,058 women in our study population, a guideline of 4 years or less was used. Among these women, $73 \%$ had had a Pap test in the last 4 years (Table 2 ). This can be compared with a compliance rate of $52 \%$ for the 2,353 women in the NHIS sample.

\section{PROSTATE CANCER SCREENING}

No recommendations are currently made for individuals to undergo prostate cancer screening by the U.S. Preventive Services Task Force. Other groups such as the American Cancer Society, the American Urological Society and the American College of Radiology recommend prostate specific antigen screening annually for men age 50 and older. ${ }^{9}$ In our study, answers of "less than one year" and "1-2 years" were used to include all men screened within the last year. Among the 50,612 males in our population, $70 \%$ reported having been screened for prostate cancer in the past 2 years (Table 2). No national comparison data were available.

\section{EMPLOYMENT STATUS AND INSURANCE COVERAGE COMPARISONS}

Compliance rates were also analyzed by gender and by insurance plan selection, either indemnity/PPO or HMO. Significantly more of those selecting HMO plans were compliant with recommended preventive services compared those in indemnity/PPO plans. There were differences in compli- ance rates between men and women with men consistently utilizing preventive services more than women (Table 2).

\section{RISK LEVEL COMPARISONS}

Among the retired individuals in our study population, $61 \%$ were categorized as low risk (0-2 risks), $27 \%$ as medium risk (3-4 risks), and $12 \%$ as high risk (5 or more risks). There were some differences in compliance with specific preventive services in the three risk level categories. Blood pressure screening by risk level was consistent with $92 \%$ compliance for all risk levels. Generally, however, there was a trend of significantly increased compliance among lower risk individuals (significant $P$-value for trend for cholesterol screening, tetanus immunization, rectal examinations and cervical cancer screening; $P<.05$ ).

Influenza immunizations were the one exception to this general trend. Compliance for flu immunizations increased significantly with increasing risk level: $69 \%$ among low-risk; $70 \%$ among medium-risk; and $71 \%$ among highrisk (significant $\mathrm{p}$-value for trend; $P<.05$ ) (Table 3 ).

\section{HRA PARTICIPATION PATTERNS}

Patterns in the usage of the HRA showed that $20 \%$ of participants completed the HRA in each of the 3 years (threetime HRA); $31 \%$ completed the HRA at least two times during the time period (two-time HRA) and 49\% completed the HRA at least once (one-time HRA). Baseline compliance rates among these three HRA subgroups indicated that participation patterns influenced preventive services compliance. For each of the preventive services considered, three-time and two-time HRA participants had higher baseline compliance than one-time HRA participants. Among one-time participants, there was no increasing trend among program years to indicate the effects of health education without participation (data not shown).

Table 3. Preventive Service Compliance by Risk Level

\begin{tabular}{|c|c|c|c|}
\hline \multirow[b]{3}{*}{ Preventive Service } & \multicolumn{3}{|c|}{ Compliance by Risk Level ${ }^{+}$} \\
\hline & $\begin{array}{l}\text { Low Risk } \\
(\mathrm{n}=36,287)\end{array}$ & $\begin{array}{l}\text { Medium Risk } \\
(\mathrm{n}=16,336)\end{array}$ & $\begin{array}{l}\text { High Risk } \\
(\mathrm{n}=7,047)\end{array}$ \\
\hline & \multicolumn{3}{|c|}{$\%$} \\
\hline Influenza immunization‡ (<1 year) & 69 & 70 & 71 \\
\hline Blood pressure screening ( $\leq 2$ years) & 92 & 92 & 92 \\
\hline Cholesterol screening ${ }^{\star}$ ( $\leq 4$ years $)$ & 85 & 82 & 79 \\
\hline Tetanus immunization ${ }^{\star}$ ( $\geq 5$ years) & 57 & 51 & 47 \\
\hline Colon cancer screening ${ }^{\star}$ ( $\leq 4$ years) & 64 & 60 & 58 \\
\hline Rectal examination ${ }^{\star}$ ( $<1$ year) & 50 & 47 & 42 \\
\hline $\begin{array}{l}\text { Breast cancer screening* ( } \leq 2 \text { years) } \\
\quad(\mathrm{n}=9,058)\end{array}$ & $\begin{aligned} & 78 \\
(\mathrm{n}= & 5,257)\end{aligned}$ & $\begin{aligned} & 75 \\
&(n=2,587)\end{aligned}$ & $\begin{array}{c}69 \\
(n=1,214)\end{array}$ \\
\hline Cervical cancer screening ${ }^{\star \ddagger}$ ( $\leq 4$ years) & 76 & 71 & 67 \\
\hline $\begin{array}{l}\left.\text { Prostate cancer screening }{ }^{*} \text { ( } \leq 2 \text { years }\right) \\
\quad(n=50,612)\end{array}$ & $\begin{aligned} & 72 \\
(\mathrm{n}= & 31,030)\end{aligned}$ & $\begin{aligned} & 69 \\
&(n=13,749)\end{aligned}$ & $\begin{array}{c}65 \\
(n=5,833)\end{array}$ \\
\hline
\end{tabular}

Note: Statistical comparisons tested for differences of compliance among low-risk, medium-risk and high-risk individuals.

${ }^{*}$ Chi-square; $P<.001$.

${ }^{\dagger}$ Overall low-risk status are those individuals who self-reported 0-2 individual risks; medium-risk, 3-4 risks; and overall high-risk, $\geq 5$ individual risks.

$\ddagger P$-value for trend $<.05$. 


\section{DISEASE STATUS AND PREVENTIVE SERVICES UTILIZATION}

Preventive service utilization and the relation to selected existing diseases (cancer, heart disease, diabetes mellitus, bronchitis/emphysema or past stroke) among the subjects in our study population were also analyzed. In this study group, $53 \%$ did not have any of the studied diseases, whereas $47 \%$ had one or more of the diseases. Among those retirees with a medical condition, $7 \%$ had cancer only, $13 \%$ had diabetes mellitus only, $9 \%$ had bronchitis/ emphysema only, $36 \%$ had heart disease only, $5 \%$ had history of prior stroke only, and $31 \%$ had multiple diseases (Table 4).

Compliance levels among the different disease categories were compared with the nondisease compliance rate. Compliance levels for influenza immunizations were higher among individuals in each disease category, including multiple diseases, when compared with individuals without disease. Individuals with cancer, diabetes mellitus, heart disease and those with multiple diseases complied with blood pressure screening guidelines at a significantly higher rate than individuals without disease. Those individuals with heart disease and multiple diseases adhered to guidelines for cholesterol screening at a higher rate compared with nondiseased individuals, whereas those with bronchitis/emphysema and prior stroke had significantly lower compliance levels. Compliance rates for colon cancer screening and rectal examinations were significantly higher among individuals with existing cancer, heart disease, and multiple diseases. Women with cancer or diabetes mellitus had a higher rate of compliance to breast cancer screening guidelines compared with women without disease. Pap screening was significantly lower among women with diabetes mellitus, heart disease, prior stroke, and multiple diseases compared with women without disease. Prostate cancer screening compliance levels were higher among men with existing cancer, heart disease and multiple diseases compared with men without disease.

\section{MULTIVARIATE LOGISTIC REGRESSION MODELS}

The multivariate regression models indicated that, generally, males, age under 70 years (with the exception of influenza immunization), HMO insurance plan selection, low-risk status, multiple HRA participants (2 or more times), and some diseases predicted increased utilization of preventive services. Selected diseases and multiple HRA participation were among the strongest predictors of increased compliance. It is noteworthy that influenza immunization, when controlled for the presence of disease in the regression model, showed an association with risk status similar to other preventive services, i.e., higher compliance among low-risk individuals (Table 5).

\section{DISCUSSION}

The HRA serves a valuable purpose in monitoring preventive services utilization among this aging population. The self-reported HRA data indicated that compliance levels within our study population were higher than national averages. In addition, the Healthy People 2000 goals for each of the preventive services studied were met and exceeded (with the exception of tetanus immunization).

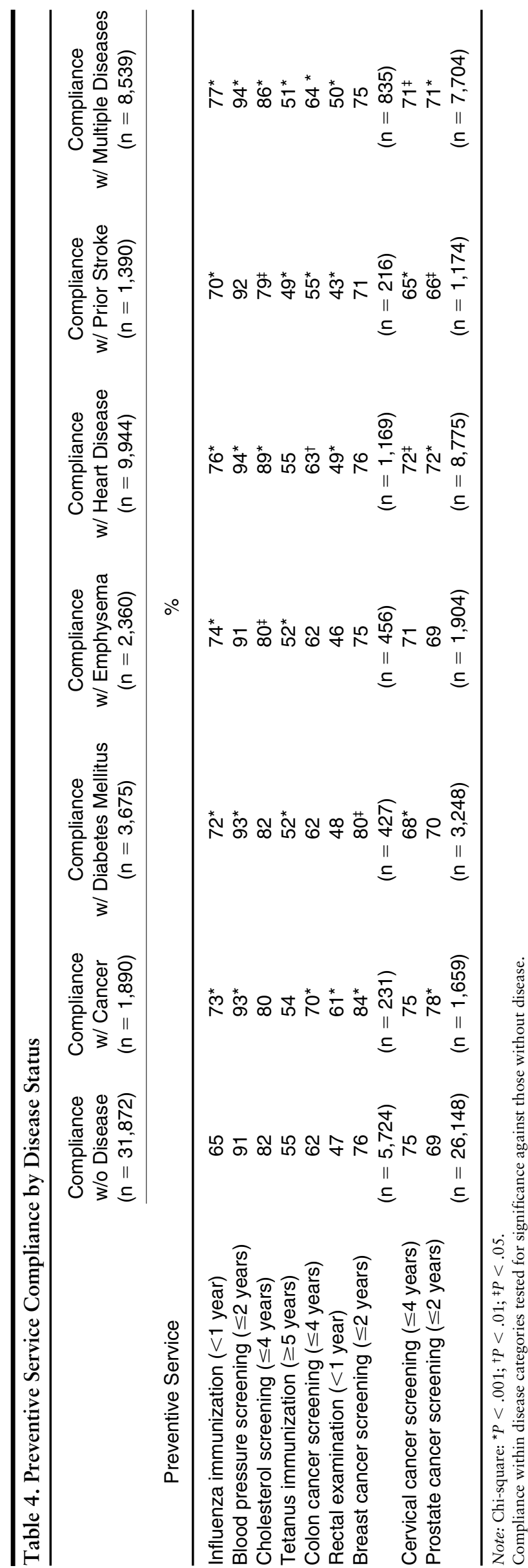


The retirees in the study were former employees of GM, which may have contributed to the observed higher compliance rates for preventive services compared with the national population. Economic status, including access to additional medical coverage, could be influential in providing opportunity for compliance. Although access to health services alone does not guarantee utilization, availability under an insurance plan removes a major barrier for retired populations. ${ }^{12-14}$ These results are consistent with previous studies, 3,5 indicating that, given awareness and opportunity, retired employees will participate at a high level in preventive health services.

Successful aging depends on many factors but increased utilization of preventive services has been associated with improved health status, decreased medical costs, specifically hospitalizations, and decreased death rates. ${ }^{3,5}$ The multivariate regression models confirmed that except for selected disease conditions, multiple HRA participation was one of the strongest predictors of increased utilization of preventive services (ORs ranging from 1.147 to 1.851). Continuing to track preventive service utilization with an HRA can provide opportunities to effectively target additional educational/awareness venues.

One important characteristic of the study group was that they participated in the HRA and received a personalized risk profile report. Although it was not possible to directly test the effect of the profile report, indirectly, increased utilization was associated with multiple year HRA participants even when risk status was controlled for in the regression models. The importance of the questionnaire and the report may be realized in increased or continued awareness of preventive service guidelines.

Controlling for other characteristics, women consistently utilized preventive services less than men (ORs of 0.523 to 0.913$)$. In the national sample, similar trends were evident. Thus the male/female compliance rates in this study group appear to be representative of general compliance trends among retired populations. These results would indicate that older women especially could benefit from targeted messages clarifying guidelines and increasing awareness of their susceptibility to selected diseases, especially heart disease and colon cancer.

In this study population, those under the age of 70 were more likely to utilize most preventive services (with the exception of influenza immunizations). Clinical Preventive Service guidelines for selected preventive services (e.g., cholesterol and breast cancer screenings) have recommended upper age limits of 65 and 69, respectively. These guidelines are focused on the cost-effectiveness of preventive services. As older adults continue to live longer with a priority of preserving quality of life and reducing disability due to disease, the upper limits for age-specific guidelines may need to be reexamined.

Health risk status (low, medium, or high risk) influences compliance rates, with generally highest compliance for preventive services among low-risk participants (with the exception of influenza immunization which increased with risk level). However, in the influenza immunization regression model, when disease conditions and other variables were controlled for, higher utilization of influenza immunization was also significantly associated with lowrisk status $(\mathrm{OR}=0.909)$. Low-risk individuals may be 
characterized by a higher health awareness that may have translated into increased compliance to guidelines.

Compliance rates for each of the selected preventive services for those employees enrolled in HMO insurance plans were significantly higher than for those enrolled in indemnity/PPO insurance plans. In the regression models, controlling for other characteristics, HMO plan selection was consistently associated with higher utilization (ORs of 1.165 to 1.389 ). Increased compliance rates may reflect increased access to preventive services in the HMO environment.

The presence of existing diseases selectively affected preventive service compliance rates. Those with any disease were more likely to get influenza immunizations. Those with cancer were more likely to comply with other cancer screenings. Those with heart disease were more likely to be screened for blood pressure and cholesterol. Because those participants with diseases are more likely to be in close contact with physicians and other medical services, this relationship is perhaps not surprising.

The HRA participant population may pose one limitation to the generalizability of these results and conclusions. One earlier study indicated that work-site wellness participants were generally healthier and more concerned with health matters than nonparticipants. ${ }^{15}$ Individuals that are more interested in their health may not only be more likely to participate in HRA activities, but also to comply with preventive service guidelines on their own. Other studies indicated no differences in health characteristics between participants and nonparticipants in health promotion activities. ${ }^{16-20}$ Previous studies on participation in preventive services by older adults indicated that the sickest and healthiest were least likely to participate. ${ }^{3,21,22}$ The cumulative HRA participation rate in this population of retired employees over the 3 years was relatively high at $39 \%$. This should minimize the potential of a self-selection bias for healthier participants or for those more likely to comply with preventive services.

A second limitation is that results were based on selfreported data and thus a recall bias may have been inadvertently introduced into the study. Reporting on preventive service utilization required that the participant remember the timeframe for the preventive service activity. However, one study reported that the accuracy of self-report influenza vaccination was $94 \%$ when validated by record review ${ }^{23}$ indicating minimal recall bias. Another more recent study confirmed that self-reports of routine screening examinations compared favorably with medical records and can provide a useful estimate for broad measures of population prevalence. ${ }^{24}$

Participation in the HRA serves a valuable purpose in monitoring the utilization of preventive services, especially in an aging population. ${ }^{14}$ The HRA can also function to promote continued and/or increased awareness of the preventive services available to this population. Increased preventive service compliance was associated with being male, younger than 70 years, multiple-year HRA participation, overall low-risk status, and HMO insurance plan selection. Compliance in selected preventive services was also associ- ated with specific diseases. Overall compliance rates among HRA participants exceeded Healthy People 2000 guidelines for all but one of the preventive services indicating a high level of utilization compared with national standards.

\section{REFERENCES}

1. Fries JF, Bloch DA, Harrington $\mathrm{H}$ et al. Two-year results of a randomized controlled trial of a health promotion program in a retiree population: The Bank of America study. Am J Med 1993;94:455-462.

2. German PS, Burton LC, Shapiro S et al. Extended coverage for preventive services for the elderly: Response and results in a demonstration population. Am J Public Health 1995;85:379-386.

3. Burton LC, Steinwachs DM, German PS et al. Preventive services for the elderly: Would coverage affect utilization and costs under Medicare? Am J Public Health 1995; 85:387-391.

4. Burton LC, German PS, Shapiro S et al. A preventive services demonstration. Health status, health behaviors, and cost outcomes 2 years after intervention. Med Care 1997;35:1149-1157.

5. Morrissey JP, Harris RP, Kincade-Norburn J et al. Medicare reimbursement for preventive care. Changes in performance of services, quality of life, and health care costs. Med Care 1995;33:315-331.

6. Vasse R, Nijhuis F, Kok G. Effectiveness of a personalized health profile for blue-collar workers. J Occup Environ Med 1998;40:69-75.

7. Boudreau R, Godin G, Pineau R et al. Health risk appraisal in an occupational setting and its impact on exercise behavior. J Occup Environ Med 1995;37:1145-1150.

8. Amonkar MM, Madhavan S, Rosenbluth SA et al. Barriers and facilitators to providing common preventive screening services in managed care settings. J Community Health 1999;24:229-47.

9. U.S. Preventive Services Task Force. Guide to Clinical Preventive Services, 2nd Ed. Baltimore, MD: Williams and Wilkins, 1996.

10. U.S. Department of Health and Human Services. National Center for Health Statistics. National Health Interview Survey, 1994: Year 2000 Objectives Supplement. Hyattsville, MD: U.S. Department of Health and Human Services, National Center for Health Statistics, 1996.

11. National Center for Health Statistics. Healthy People 2000 Review, 199899. Hyattsville, MD: National Center for Health Statistics, 1998.

12. Powell-Griner E, Bolen J, Bland, S. Health care coverage and use of preventive services among the near elderly in the United States. Am J Public Health 1999;89:882-886

13. McCall N, Rice T, Boismier J et al. Private health insurance and medical care utilization: Evidence from medicare population. Inquiry 1991;28:276-287.

14. CDC. Surveillance for use of preventive health-care services by older adults, 1995-1997. MMWR Morb Mortal Wkly Rep 1999;48:51-88.

15. Conrad P. Who comes to work-site wellness programs? A preliminary review. J Occup Med 1987;29:317-320.

16. Stange K, Strogatz D, Schoenback VJ et al. Demographic and health characteristics of participants and nonparticipants in a work site health-promotion program. J Occup Med 1991;33:474-478.

17. Grosch JW, Alterman T, Petersen MR et al. Worksite health promotion programs in the U.S.: Factors associated with availability and participation. Am J Health Promot 1998;13:36-45.

18. Klesges RC, Wiliamson JE, Somes GW et al. A population comparison of participants and non-participants in a health survey. Am J Public Health 1999;89:1228-31.

19. Wagner EH, Grothaus LC, Hecht JA et al. Factors associated with participation in a senior health promotion program. Gerontologist 1991;31:598-602.

20. Buchner DM, Pearson DC. Factors associated with participation in a community senior health promotion program: A pilot study. Am J Public Health 1989;79:775-777.

21. Ives DG, Traven ND, Kuller LH et al. Selection bias and nonresponse to health promotion in older adults. Epidemiology 1994;5:456-461.

22. Schweitzer SO, Mayer-Oakes SA, Atchison KA et al. Health promotion and disease prevention for older adults: Opportunity for change or preaching to the converted? Am J Prev Med 1994;10:223-229.

23. Hutchison BG. Measurement of influenza vaccination status of the elderly by mailed questionnaire: Response rate, validity, and cost. Can J Public Health 1989;80:271-275.

24. Martin LM, Leff M, Calonge $\mathrm{N}$ et al. Validation of self-reported chronic conditions and health services in a managed care population. Am J Prevent Med $2000 ; 18: 215-218$ 